\title{
Sleep disorder detection and identification
}

\author{
Dennis E. B. Tan, Wai Yie Leong \\ Universiti Tunku Abdul Rahman (UTAR), Kuala Lumpur, Malaysia \\ Email: milktea1226@hotmail.com,waiyie@ieee.org
}

Received January $17^{\text {th }}, 2012$; revised February $17^{\text {th }}, 2012$; accepted March $2^{\text {nd }}, 2012$

\begin{abstract}
Electroencephalogram (EEG) is one of the medical devices that used for sleep disorder detection. Sleep disorder such as Obstructive Sleep Apnea Syndrome (OSAS) often appears during sleep event. Since the OSAS patients have the difficulties to allow the airflow into the lung while inspiration, the EEG is applied to capture and record the brainwave of the patient. In this work, the Empirical Mode Decomposition (EMD) and Ensemble Empirical Mode Decomposition (EEMD) are used to process and analyze the accuracy and efficiency of the results. Both of these methods will decompose the EEG signal into a collection of Intrinsic Mode Function (IMF). In this paper, index orthogonality has been calculated to indicate the completeness of the decomposed signal with the original signal. The instantaneous frequency and Hilbert Spectrum based on both methods also employed by IMF to analyze and present the results in frequency-time distribution to determine the characteristic of the inherent properties of signal. Besides, Hilbert marginal spectrum has been applied to measure the total amplitude contribution from each frequency value. Finally, the results shown that the EEMD is better in solving mode mixing problem and better improvement over EMD method.
\end{abstract}

Keywords: Empirical Mode Decomposition (EMD); Intrinsic Mode Function (IMF); Ensemble Empirical Mode Decomposition (EEMD); Hilbert Spectrum

\section{INTRODUCTION}

Obstructive Sleep Apnea Syndrome (OSAS) is a common sleep disorder problem that always happens among the general population nowadays. According to the [1], Wisconsin Sleep Cohort which is a comprehensive epidemiologic study investigating of OSAS in middle-aged adults, found that $4 \%$ of men and $2 \%$ of women has OSAS. The sleep disorder occurs when the collapse of throat's tissue which leads to partially or completely blockage of airways flow into lung during inspiration. The OSAS will seriously affect the daily activities of the patients who suffer from sleep disorder. Daytime sleepiness and the likelihood to sleep during driving are the most common negative effects. Besides, their health will also be affected due to lack of sleeping time which may cause high blood pressure, stroke, abnormal heart rhythms and even death due to myocardial infarction.

From the observation on OSAS patients, the most common symptoms are choking or gasping at the termination of obstructive apnea and this will cause selfarousal. From here, the electroencephalogram (EEG) can be used as a medical device to carry out the analysis. In order to carry out the diagnosis and interpretation proposed by the healthcare provider, the (EEG) has been used widely to capture and collect the human brainwave. Although the signal has been captured by EEG, processing these data accurately and efficiently are always the key problem for most of the scientists. The signals which performed by EEG contains of noise from the environment although the embedded system is established in EEG devices. Data analysis becomes necessary in research and practical application in medical field. By ensuring the accuracy in signal processing and feature extraction, the analysis of data can be done well and also capable of providing appropriate solutions. The EEG is a non-stationary and non-linear signal with amplitude, phase and frequency continuous changes [2]. The EEG signal can be analyzed by any kind of time-frequency analysis methods [2].

In this work, the recorded EEG signal will be processed into more useful information to analysis OSAS. One well-established information fusion model, namely the waterfall model is shown in Figure 1. There are several processing stages, starting from the raw data acquisition to situation assessment and decision making stages [3]. Decision making stage can be achieved by inspection or by post-processing in the form of machine learning algorithms such as Support Vector Machines (SVM) which proposed by [4]. In this paper, signal processing and feature extraction will be the main focus in order to enhance the accuracy of data analysis. There are plenty of methods to analyze signals such as Fourier analysis, Wavelet analysis, Independent Component Analysis (ICA), etc. In general, the data which need to be analyzed have 


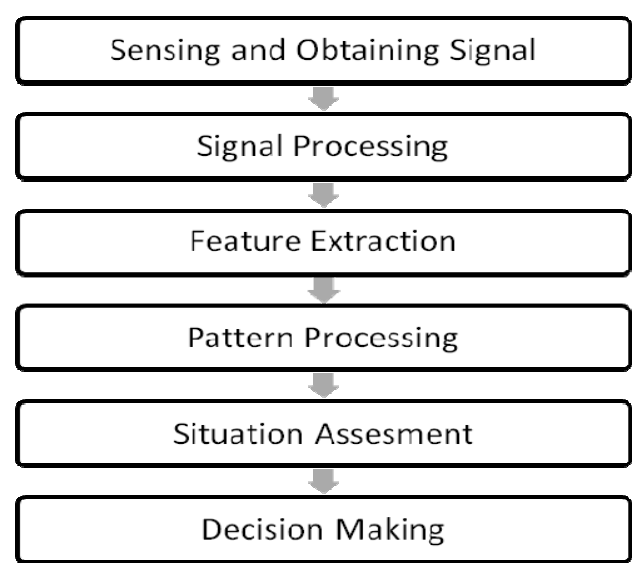

Figure 1. The "waterfall model" of information fusion.

several problems. For example, lack of total data span; data are non-stationary and non-linear processes. However, two solutions which are the Empirical Mode Decomposition (EMD) and the modified original EMD so called Ensemble Empirical Mode Decomposition (EEMD) will be emphasized in this paper. Hilbert Spectral Analysis also will be applied onto the Intrinsic Mode Functions (IMFs) to analyze the useful information. The EEG signal processing based on EEMD has been proposed by [5] which provides new bases for feature extraction and pattern recognition of EEG signals.

EMD has been proposed by [6] as an adaptive timefrequency data analysis method. This method has been proven high capability for extracting signals in a broad range of applications. EMD decomposes the data into a set of Intrinsic Mode Function (IMF) components, which become basis representing the data. These data can be further presented through Hilbert Spectral Analysis. The obtained IMF components can be used in applying Hilbert transform onto each of them. Since the basis is adaptive, there is no need for harmonics [7]. Therefore, EMD is a method for analyzing nonlinear and non-stationary and it is an alternative to the wavelet analysis and the shorttime Fourier Transform [8]. However, there is still remaining deficiency in the decomposed data.

The major drawback of EMD is the frequent appearance of mode mixing. This is the consequence of signal intermittency and affects the physical meaning of IMF components. The intermittence will cause serious aliasing in the time-frequency distribution [6]. To improve the efficiency of original EMD, the application of noiseassisted data analysis (NADA) method, so called Ensemble Empirical Mode Decomposition (EEMD) which discussed before by [9] is necessary. EEMD is applied to solve the mode mixing problem through the decomposition of EEG signal into IMF components. Hilbert spectrum can act as a tool to observe and analysis the comparison between IMFs of these two EMD and EEMD.

\section{METHODOLOGY}

In this paper, the data set was created and contributed by the developers of the BCI2000 instrument system and downloaded from the PhysioNet. These data was used as the simulation object. These data whose sampling frequency is $100 \mathrm{~Hz}$ had been collected by EEG from a severe OSAS patient. The EEG electrodes have been used to collect brain signal, the other medical devices are used to derive extra information and conditions from patient during sleep. For example, electrooculogram (EOG) to measure resting potential of retina; electromyogram (EMG) to evaluate and record the electrical activity produced by skeletal muscles; electrocardiogram (ECG) to measure the electrical activity of heart, etc. Since there are a lot of data from various medical devices, only the EEG data will be chosen and focused in our simulation and discussion. These EEG data will be processed and analyzed through EMD method and EEMD method by decomposing the data into IMF that admit well-behaved Hilbert transform. Hilbert Spectrum will aid to apply for general purpose in energy-frequency-time distribution such as identify the basic frequency and their range variation.

\subsection{Empirical Mode Decomposition (EMD)}

Any complicated data set can be adaptively decomposed into IMF components through EMD method. The term IMFs, are the functions that need to satisfy two conditions [6]:

1) In the whole time series, the number of extrema and the number of zero crossings must be either equal or differ at most by one.

2) At any point in the time series, the mean value of the envelopes which is defined by local maxima (upper envelope) and local minima (lower envelope) is equal to zero.

EMD method is a sifting process that estimates IMFs. The "stopping criterion" actually determines the number of sifting steps to produce IMF [10]. The envelopes are defined by local maxima and local minima. When these extrema are identified, all the local maxima and local minima will be connected by spline line as upper envelope and lower envelope which should cover all the data between them respectively. The mean is designated as $m_{1}$ and the difference between original data, $x(t)$ and $m_{1}$ is the first component, $h_{1}$ as shown:

$$
h_{1}=x(t)-m_{1}
$$

The purposes of sifting process are eliminating the riding wave and produce more symmetrical wave-profile [3]. Therefore, the sifting process is required to repeat several times. For the second sifting process, $h_{1}$ is treated as the data: 


$$
h_{11}=h_{1}-m_{11}
$$

Sifting process will be continued up to $k$ times when $h_{1 k}$ is fulfilling the two conditions of IMF to become the first IMF component, $c_{1}$ :

$$
h_{1 k}=h_{1(k-1)}-m_{1 k}
$$

where

$$
c_{1}=h_{1 k}
$$

Determination of stopping criterion for the sifting process is necessary to ensure the IMF components contain enough physical sense of both amplitude and frequency modulations. Hence, the standard deviation, SD, criteria is applied by limiting its size:

$$
S D=\sum_{t=0}^{T}\left[\frac{\left|\left(h_{1(k-1)}(t)-h_{1 k}(t)\right)\right|^{2}}{h_{1(k-1)}^{2}(t)}\right]
$$

The typical value of SD can be set between 0.2 and 0.3 . The original signal, $x(t)$ is then separated with the first IMF component, $c_{1}$ through

$$
x(t)-c_{1}=r_{1}
$$

Residue $r_{1}$ is taking as new data since it still contains information of longer period components. The process will be repeated:

$$
\begin{aligned}
& r_{1}-c_{1}=r_{2} \\
& r_{n-1}-c_{n}=r_{n}
\end{aligned}
$$

Finally, Eq.6 and Eq.7 are summed up and expressed as the summation of all the IMF components and the residue signal:

$$
X(t)=\sum_{i=1}^{n} c_{i}+r_{n}
$$

\subsection{Ensemble Empirical Mode Decomposition (EEMD)}

As mentioned before, the major drawback of EMD is the effect of mode mixing. To overcome this problem, the EEMD which is the improved EMD method has been applied in this work. When the signal is intermittent, means not continuous, will result as affecting the characteristic component of different time scales, an IMF component can increase their physical meaning of the original signal since the interruption of signal is occurred to perturb the time-frequency distribution. The EEMD defines the true IMF components as the mean of an ensemble of trials, each consisting of the signal plus a white noise of finite amplitude. The added white noise would populate the whole time-frequency space uniformly, facilitating a natural separation of the frequency scales that alleviate the exist of mode mixing [4]. The collection of white noise will cancel each other in a time space ensemble mean, so only the signal can survive in the final noise-added signal ensemble mean [9]. According to [5], two situations will be shown as the mode mixing exists, which are:

1) A single IMF component of the signal contains various components of scales;

2) Same components exist in different scales of IMF.

This NADA method, so called EEMD is developed as follows [5,9]:

1) A white noise series is added into the signal,

$$
X_{i}(t)=x(t)+w_{i}(t)
$$

where $w_{i}(t)$ is $i^{\text {th }}$ copies of white noise.

2) The added white noise's signal is processed to decompose into IMF components.

3) Step 1 and step 2 are repeated with different white noise series each time.

4) Obtain the (ensemble) means of corresponding IMFs of the decomposition as the final result.

$$
c_{j}(t)=\lim _{N \rightarrow \infty} \frac{1}{N} \sum_{k-1}^{N} C_{j k}(t)
$$

\subsection{Index Orthogonality}

Theoretically, all of the decomposed IMF components should reconstruct the original data set to indicate that they are orthogonal to each other [11]. It is used to ensure the completeness and orthogonality of this decomposition. According to the [6], they define an index based on the most intuitive way to check the orthogonality of the IMFs from EMD and EEMD as following:

1) Rewrite Eq.8 as

$$
X(t)=\sum_{j=1}^{n+1} c_{j}(t)
$$

In which the $r_{n}$ is included as an additional element.

2) Form the square of the signal

$$
X^{2}(t)=\sum_{j=1}^{n+1} c_{j}^{2}(t)+2 \sum_{j=1}^{n+1} \sum_{k=1}^{n+1} c_{j}(t) c_{k}(t)
$$

3) With this expression, the overall index of orthogonality, IO, is defined as

$$
\mathrm{IO}=\sum_{t=0}^{T}\left(\sum_{j=1}^{n+1} \sum_{k=1}^{n+1} c_{j}(t) c_{k}(t) / X^{2}(t)\right)
$$

However, the EMD method which proposed by [6] is not fully fulfilling the theory of the orthogonality toward these IMF. The index of orthogonality is depending on the amount of leakage when decomposing the signal. The leakage which will affect the index of orthogonality of the decomposed data from the original data should be as low as possible to enhance the analysis result. The IMFs of EEMD method will be compared with EMD method to show their performance later. 


\subsection{Hilbert Spectral Analysis}

Hilbert Transform will be applied onto each of the IMF components after decomposition had been done. Through Hilbert transform, obtaining the instantaneous frequency of each IMF will have no difficult to analysis the result on Hilbert Spectrum. The instantaneous frequency of the original signal is defined as [12].

$$
w_{j}(t)=\frac{\mathrm{d} \theta_{j}(t)}{\mathrm{d} t}
$$

where $\theta_{j}(t)$ is instantaneous phase.

After performing the Hilbert-Huang transform (HHT), we can express the data in the following form [12]:

$$
X(t)=R_{e} \sum_{i=1}^{n} a_{j}(t) \exp \left(i \int w_{j}(t) \mathrm{d} t\right)
$$

where $a_{j}(t)$ is the instantaneous amplitude.

Since the Hilbert spectrum has been defined, the Hilbert marginal spectrum is shown as following:

$$
h(w)=\int_{0}^{T} H(w, t) \mathrm{d} t
$$

where $T$ is the total data length. Hilbert marginal spectrum is the time integration of Hilbert spectrum, $H(w, t)$ which is a reduced frequency-energy representation [10].

\section{SIMULATION RESULTS AND DISCUSSION}

The complete set of the original EEG data are collected from severe OSAS patient which is called as objects contain of 120,000 samples for each of the channels and sampling frequency is $100 \mathrm{~Hz}$ for 20 minutes. These EEG data in Figure 2 corresponds to channels C3, C4, $\mathrm{P} 2, \mathrm{Pz}, \mathrm{O} 1, \mathrm{O} 2$ and reference point (mastoid area) according to the 10 - 20 EEG electrodes placement. Since there are several channels of EEG signals, the electrode which is placed on the C3 channel will be chosen for analysis based on EMD, EEMD and Hilbert transform. The C3 location is related to the electrical activity in somato-sensoric and motoric brain areas [13]. Due to the amount of time samples of C3 is large, the time samples between $91,500^{\text {th }}$ and $93,000^{\text {th }}$, which is totally 1500 samples as in Figure 3 will be observed and analyzed.

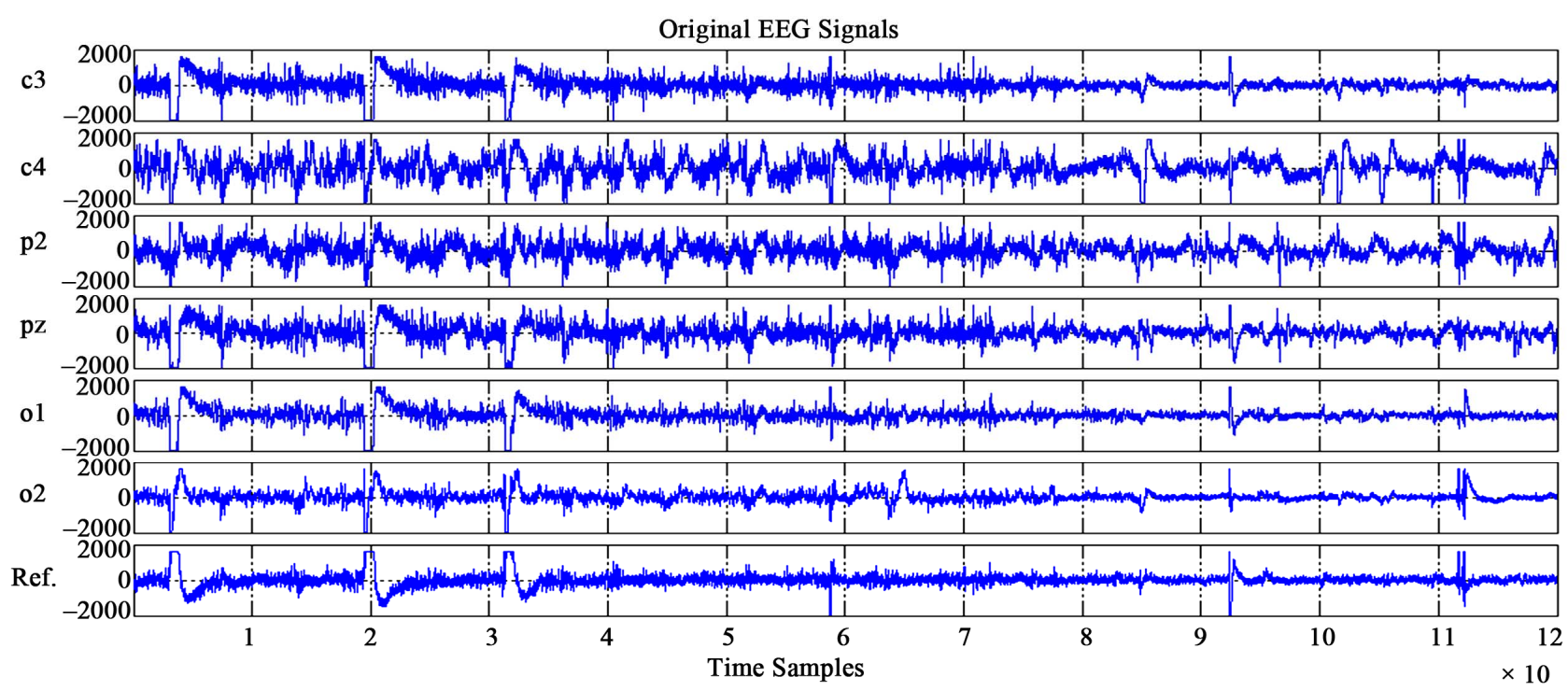

Figure 2. The original EEG data from severe OSAS patient.

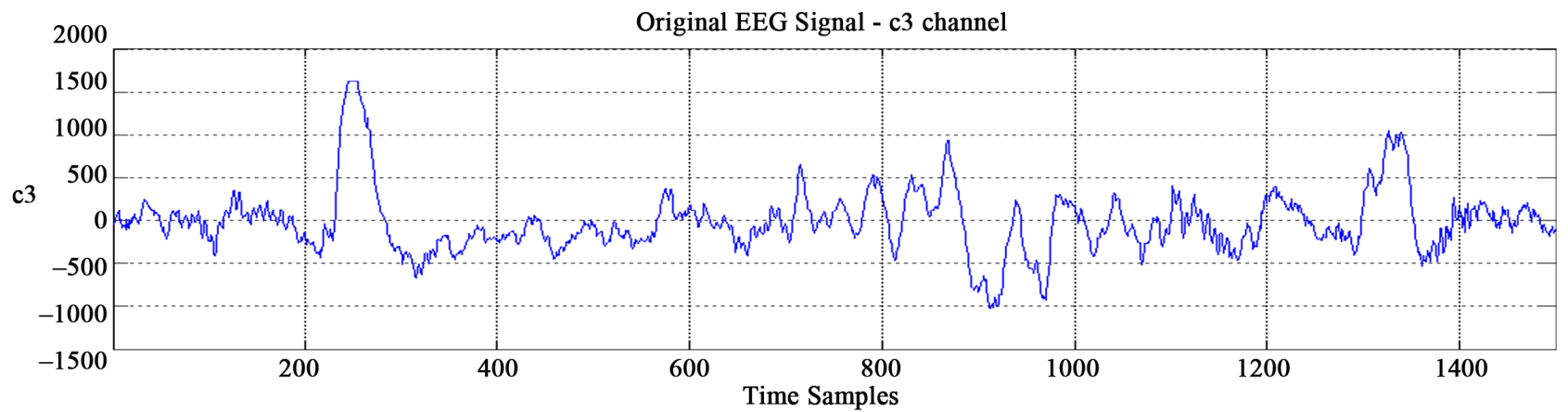

Figure 3. The original 1500 samples EEG data from C3 channel of severe OSAS patient. 
The original C3 channel EEG signal in Figure 3 has been decomposed by EMD and EEMD respectively. These IMFs are based on EMD in Figure 4 and EEMD in Figure 5 have been arranged from low to high order. The EEMD is implemented with the added noise having amplitude of 0.1 standard deviation in C3 channel. With the observation onto these IMFs, the frequency of each individual IMF decreases as the order of IMF increases. Overall, the decomposition of EEMD is more precise and accurate and the waveform presented is regular compared to EMD. We can observe that the EEMD method still retains more useful information. This happens significantly by comparing the third IMF of EMD and EEMD methods. Obviously, from samples $600^{\text {th }}$ to $1000^{\text {th }}$, the third IMF based EMD contains less physical meanings compared to the EEMD method.

From the decomposed signals through observation, how can we ensure that these IMFs whether had established the completeness of the decomposition or the dif- ference between the reconstructed data from the sum of all IMFs and the original data were exists? One of the methods to solve this question is to check the orthogonality of IMF components which had been proposed by [14]. The index of orthogonality can be calculated and the interpolation techniques can be evaluated from the According to the [6], the orthogonality is required in linear decomposition systems; it might not make physical sense for nonlinear decomposition as in EMD and EEMD. But, in most cases, the leakage is still considered small. However, we still can compare the index of orthogonality (IO) in both methods since the data is the same from C3 channel. Table 1 shows the IO of EMD

Table 1. Comparison of IO values between EMD and EEMD methods.

\begin{tabular}{cc}
\hline Decomposition method & Index of orthogonality (IO) \\
\hline EMD & 0.2579 \\
EEMD & 0.1989 \\
\hline
\end{tabular}

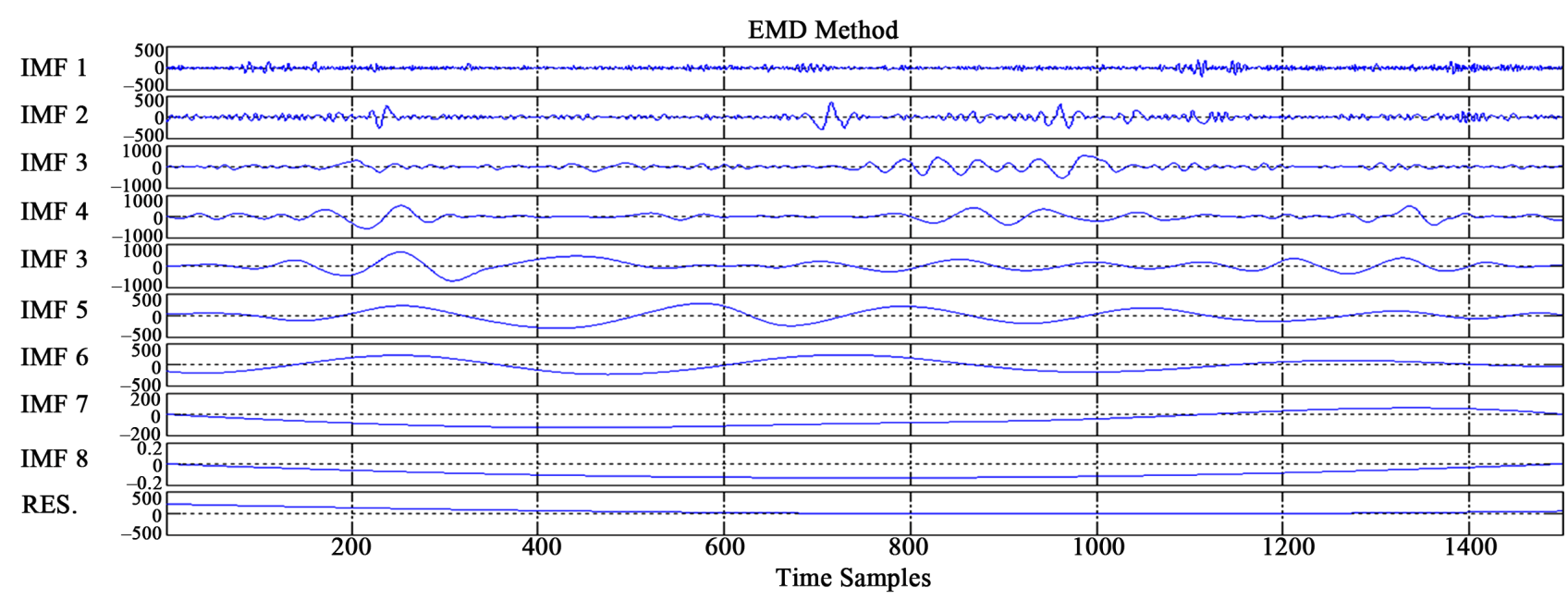

Figure 4. EMD method.

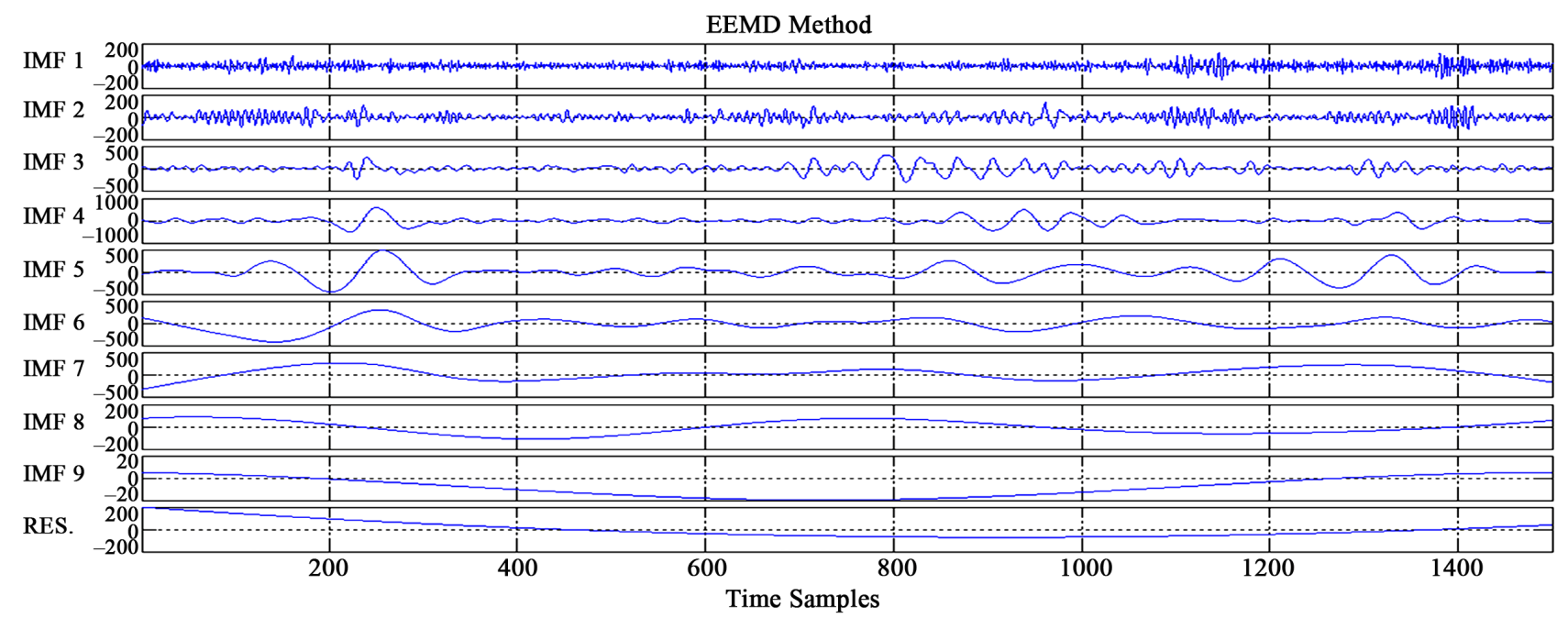

Figure 5. EEMD method. 
and EEMD respectively. These values indicate the leakage level of the decomposed signal. Observed that the IO values are "less orthogonal" to EMD and EEMD, but these will still provide us a result to determine a better method. From table 1, it shows that IO value based on EMD method which is 0.2579 is quite higher much compare with the EEMD method which is 0.1989. As low as the IO value, the difference between the reconstructed data from sum of all IMFs component and the original data is very small. Another word, the leakage while decomposition of signals is less as the IO value is very low. From here, we can confirm that the EEMD method can retain more physical meaning and less leakage of information will be loss on the decomposed signals, which are IMF components.

Next, we can do some comparison of the details of IMF components for each method by observation. Before analyzed these decomposed signals, there are a few properties of original EMD is presented according to the [9]. The main properties are the dyadic property always compromised in the original EMD while the data is intermittent. Therefore, the purpose of adding noise is to provide a uniformly distributed reference scale. This will enable EMD to solve the problem of compromised dyadic property. Since the different series of noise which corresponding to the IMFs have no correlation with each other, the mean of different white noise series will cancel each other. The comparison between original signal with IMF components based on EMD and EEMD had been carried out to show their difference in containing the useful information in decomposed data. In this paper, the fifth IMF component (for EMD is Figure 6, EEMD is Figure 7) and sixth IMF component (for EMD is Figure 8, EEMD is Figure 9) were selected to analyze and compare to the original C3 channel EEG data which shows in Figure 3.

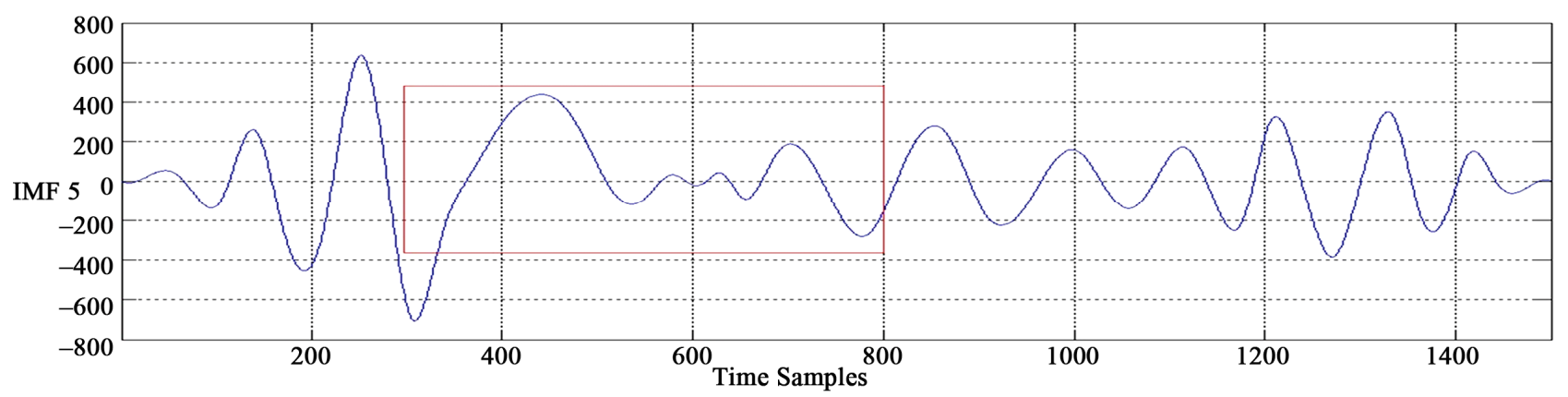

Figure 6. IMF5 of EMD.

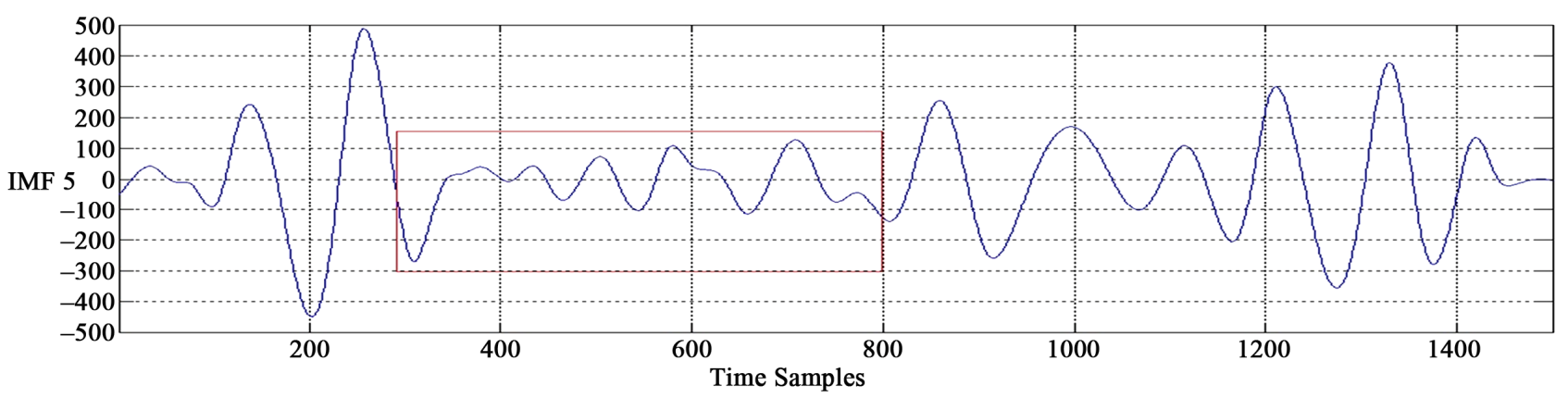

Figure 7. IMF5 of EEMD.

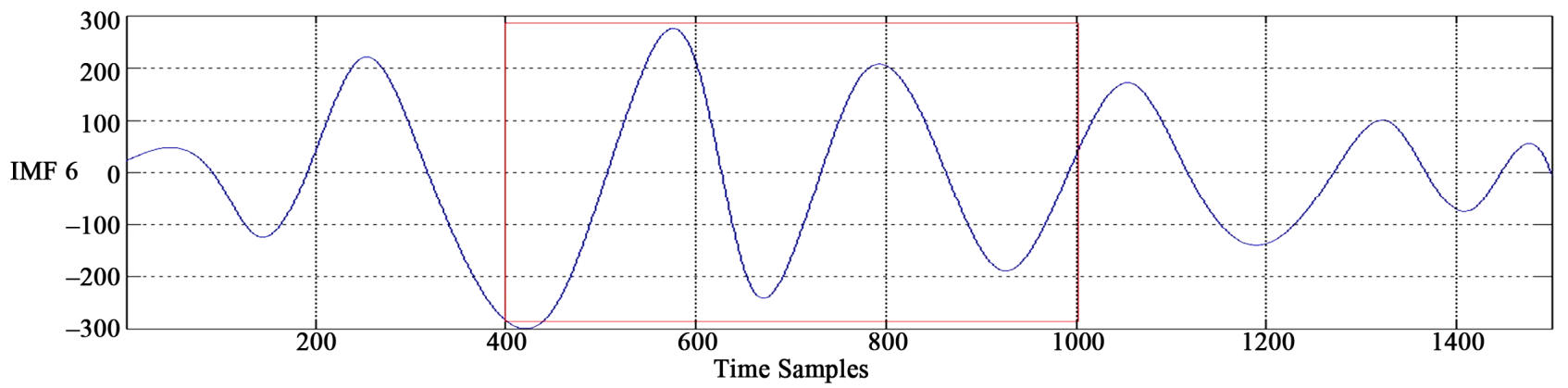

Figure 8. IMF6 of EMD. 
Through the observation of C3 channel EEG signal, we can briefly know that between the $200^{\text {th }}$ to $300^{\text {th }}$ samples, the event of sleep apnea was occurred. From Figures 6 to 9, the spike within these hundred samples range is still remained after applying EMD and EEMD process. So, we can ensure that the expectation is realistic. Compare with the C3 EEG data, the fifth IMF of EEMD in red column is obviously containing more information compare to the EMD as shows in the figure.

Same condition appears in the sixth IMF of EEMD and EMD. As the decomposition process go along to the higher order, the EMD is unable to avoid the mode mixing problem since the dyadic property is comprised which it had loss the physical meaning of the data. Conversely, EEMD still able to retain more information since the added white noise had solved this problem. The decomposition of EEMD had proved that it can present well the inherent physical properties of the original signal. Therefore, the EEMD is enabling EMD to repair the compromised dyadic property which can regulate the mode mixing problem effectively.

After obtaining the IMF components of EMD and EEMD, the Hilbert transform can be applied to construct the time-frequency-energy distribution, designated as Hilbert Spectrum [15]. Hilbert spectra analysis can be used in exploring the full physical meanings of the complicated data such as EEG data we analyzed here. Hilbert spectrum can perform the high time-frequency resolution; the signal can characterize the local features of the signal effectively by combining the instantaneous spectrum of each IMF component [5]. The Hilbert-Huang spectrum is shown in Figures 10 and 11.

According to [9], Hilbert spectra can be used to identify the basis frequency and ranges of variation but not in quantitative measurement. Although it is realistic, we still know that EEMD method shows good improvement in Hilbert spectrum. As we can see in Figures 10 and 11, we can observe that the comprehensible and unmistakable were appeared on the characteristics of Hilbert spectrum based on EEMD compare to the EMD. From here, we can confirm that the accuracy and efficiency of the EEMD method are better compare with the EMD. For Figure 10, the time-frequency distribution has been affected and the trace was separated from one scale to another in certain distance due to the mode mixing effect in EMD method. Conversely, in Figure 11, EEMD method has alleviated the problem of mode mixing since there are less or almost no transitional gap occur. The continuous basis frequency also indicated that the inherent physical information can be retained and handled so that the problem of mode mixing can be ameliorated.

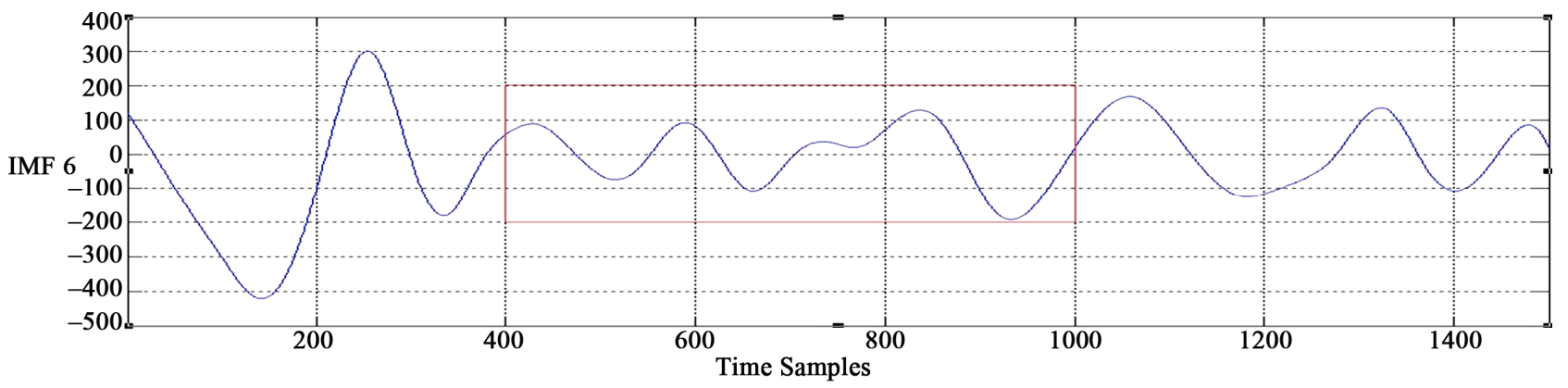

Figure 9. IMF6 of EEMD.

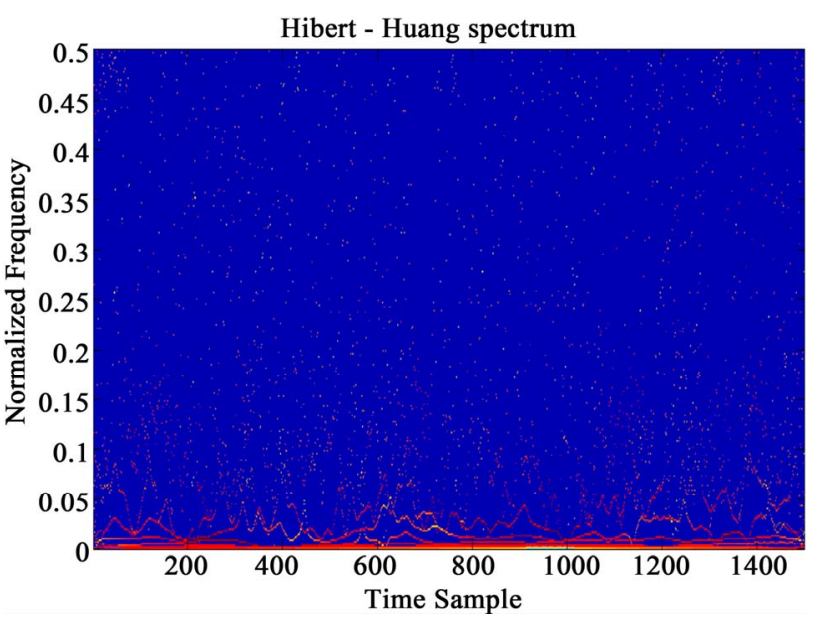

Figure 10. Hilbert spectrum of EMD method.

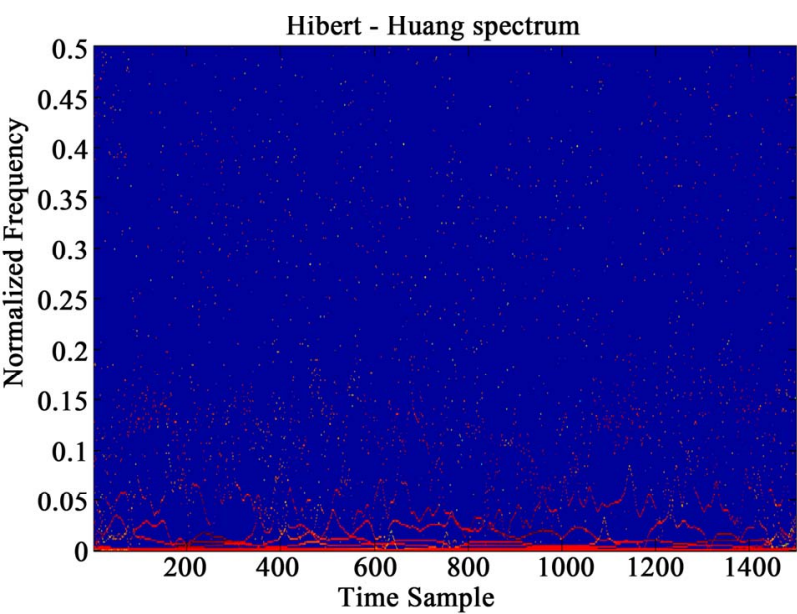

Figure 11. Hilbert spectrum of EEMD method. 
According to [16], the instantaneous frequency is employed to understand the detailed mechanisms of nonlinear and non-stationary signal. Through the Hilbert transform, the instantaneous frequency also can derive to compare the accuracy of the result based on these two methods. Instantaneous frequency can be calculated by Equation (14) where it is mentioned before. The instantaneous frequency of EEG signals based on EMD and EEMD methods has been shown in Figures 12 and 13. Observed that the red colour line which is IMF 3, the mode mixing was appeared obviously from the time samples $500^{\text {th }}$ to $1000^{\text {th }}$ based on EMD compared to the
EEMD. The instantaneous frequency based EEMD, at the same ranges of time samples provided more information to analyze the EEG signal.

Lastly, Hilbert marginal spectrum will be presented to describe the total amplitude (or energy) contribution of OSAS signal from each frequency value. Figures 14 and 15 shown the Hilbert marginal spectrum obtained by EMD and EEMD methods. Through the graphs, we can observe that the characteristics of OSAS's EEG signal are more obvious in marginal spectrum by EEMD compared to the EMD. Therefore, the severity and condition of OSAS event can be determined.

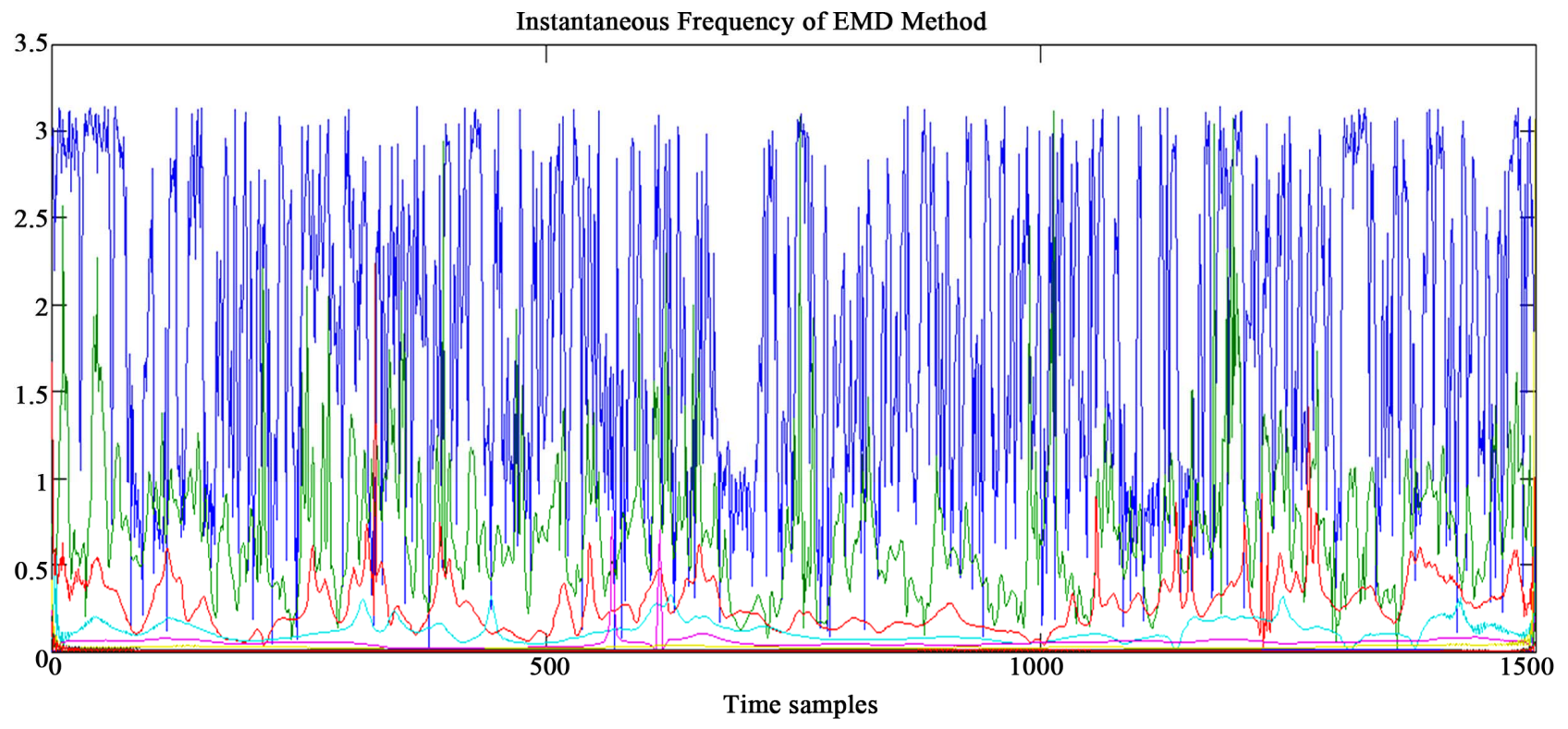

Figure 12. Instantaneous frequency of EMD method.

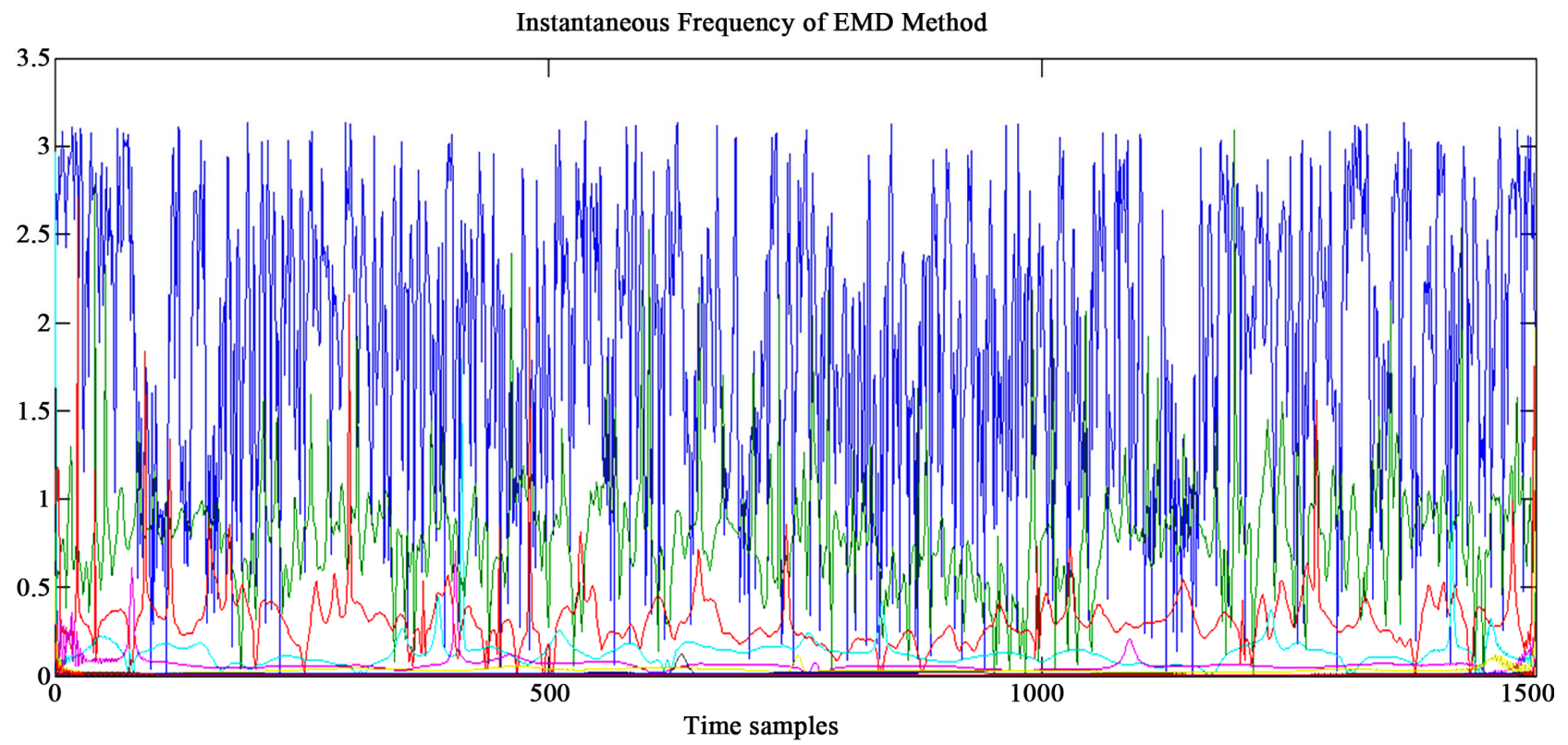

Figure 13. Instantaneous frequency of EEMD method. 


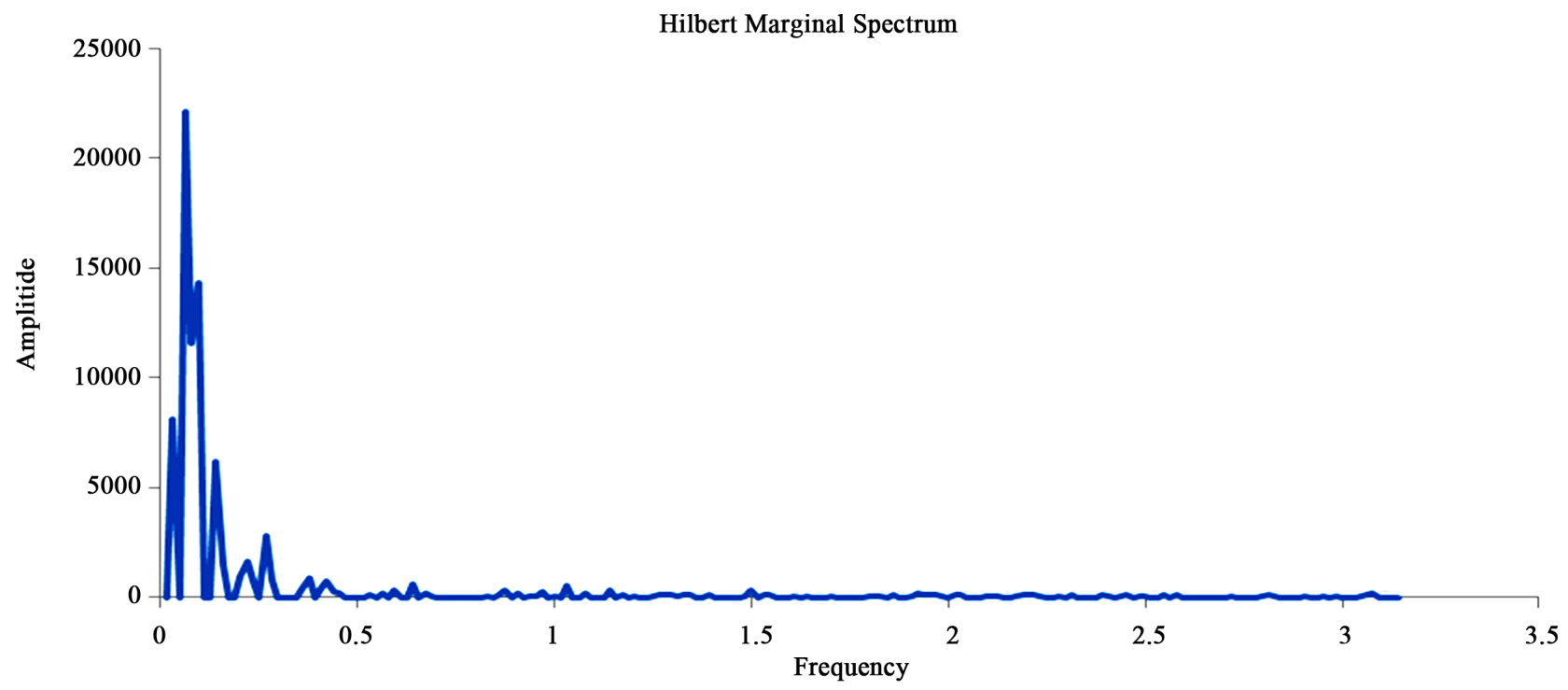

Figure 14. Hilbert marginal spectrum by EMD method.

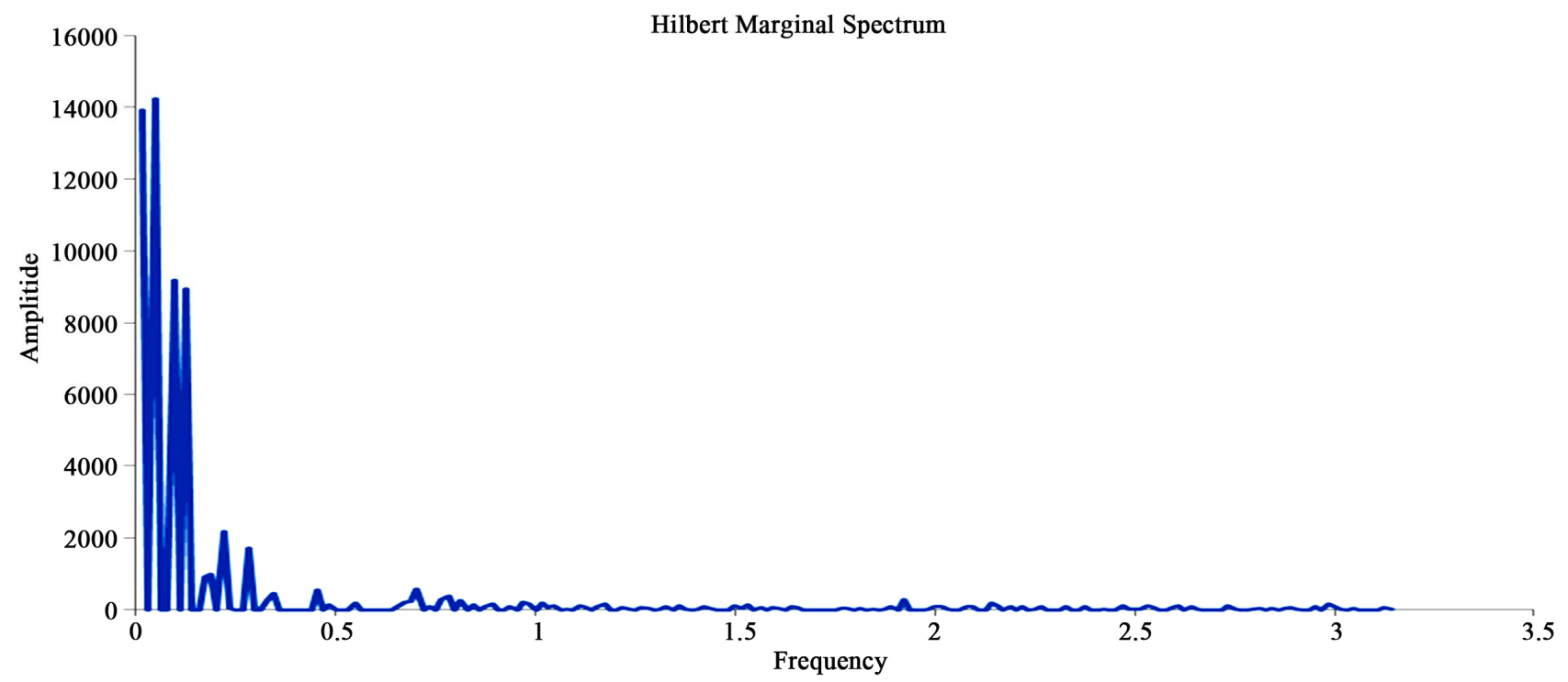

Figure 15. Hilbert marginal spectrum by EEMD method.

\section{LIMITATIONS AND CHALLENGES}

In this paper, the limitation of EMD method has been resolved using the improved EEMD method. The separation of signals of different scales by EEMD has successfully improved the result by reducing the effect of mixing mode. The most important thing is, the EEMD has cancelled off the background noise since it is zero mean of noise after these noise acts as the provider to distribute uniformly frame of scales. With this technique, the physical meaning of the inherent properties of signals can be retained for analysis purpose.

Although the EEMD has been proven a good improvement of the EMD method, there are still remaining drawbacks [9]. Handling the multi-mode distribution of the IMF components is the main drawback of EEMD. Referring to [9], the problem appeared due to the overlapping of scales in dyadic filter which is based on [7]. Two different modes will occur due to the signals where having a scale located in overlapping region. For bimodal, one of the alternative ways is the number of sifting times must be low to derive these IMF components. Another one is the noise level which has been adjusted and increased the number of trials to decrease root-meansquare deviation. However, handling of multi-mode distribution is still remained problem although these methods have been carried out.

Recently, multivariate EMD has been proposed [17] by Rehman \& Mandic to alleviate the problem in originnal EMD algorithm which is the computation of the local 
mean of the original signal. The standard EMD method lacks of capability to process the complex signals. Multivariate extensions of EMD show the improvement over EMD for bivariate [3] and trivariate [18] processing. Since the original EMD has the limitation in analyzing the bivariate data, the rotation-invariant EMD (RI-EMD) is first proposed by Altaf et al. [19] to obtain the local mean of bivariate signal by using component-wise spline interpolation and then averaging the local extrema. For the extension EMD for trivariate signal, the equi-longitudinal lines is used to produce a set of multiple direction vectors in $3 \mathrm{D}$ to project onto the trivariate signal for estimation of local mean and envelopes. Similarly, the multivariate EMD algorithm employed the concept of bivariate and trivariate as it also taking signal projections along different directions in $n$-dimensional spaces to produce multiple $n$-dimensional envelopes and the further averaged them to derive the local mean. Further study will be carried out in this field later.

\section{CONCLUSION}

This paper investigated the EMD and EEMD methods for EEG signal from OSAS patients. The EEMD applied NADA method, the added noise provided uniform distribution onto the signal. The zero-mean characteristics has resulted the noise cancelling out each other. Finally, the mean value of IMF will be taken as a result. These results were presented through Hilbert spectrum and Hilbert marginal spectrum in frequency-time distribution and amplitude (or energy)-frequency distribution. Both of them show that the EEMD has better performance compared to the EMD. It can be concluded that the EEMD method has the capability to eliminate the mode mixing problem from the original EMD method. It is important to avoid data losing features during the decomposition process and ensure the physical meaning of inherent properties of the signals was retained. This useful information provides an idea to extract the feature and recognize the pattern of OSAS's EEG signal for diagnosis and treatment purposes.

\section{REFERENCES}

[1] Lee, E.-K. and Jerri, R.H. (2001) Sleep disorder. Sacramento, Greenville.

[2] Yi, L., Fan, Y.-L., Li, G. and Tong, Q.-Y. (2009) Sleep stage classification based on EEG hilbert-huang transform. 4th IEEE Conference on Industrial Electronics and Applications, Xi'an, 25-27 May 2009, 3676-3681.

[3] Mandic, D.P., Souretis, G., Leong, W.-Y., Looney, D., Van Hulle, M.M. and Tanaka, T. (2008) Complex empirical mode decomposition for multichannel information fusion. In: Mandic, D., Golz, M., Kuh, A., Obradovic, D. and Tanaka, T., Eds., Signal Processing Techniques for
Knowledge Extraction and Information Fusion, Springer, New York, 243-249. doi:10.1007/978-0-387-74367-7 13

[4] Bao, Y.-K., Zhang, R., Xiong, T. and Hu, Z.-Y. (2001) Forecasting non-normal demand by support vector machines with ensemble empirical mode decomposition. Advances in Information Sciences and Service Sciences, 3, 81-91.

[5] Zhu, X.-J., Lv, S.-Q., Fan, L.-J. and Yu, X.-L. (2011) The EEG signal process based on EEMD. IEEE International Symposium on Intelligent Processing and Trusted Computing, Hubei, 22-23 October 2011, 222-225. doi:10.1109/IPTC.2011.67

[6] Huang, N.E., Shen, Z., Long, S.R., Wu, M.C., Shih, H.H., Zheng, Q., Yen, N.-C., Tung, C.C. and Liu, H.H. (1998) The empirical mode decomposition and the hilbert spectrum for nonlinear and non-stationary time series analysis. Proceeding Royal Society London, 454, 903-995.

[7] Wu, Z.-H. and Huang, N.E. (2004) A study of the charac- teristics of white noise using the empirical mode decomposition. Proceeding Royal Society London, 460, 1597- 1611.

[8] Demir, B. and Erturk, S. (2008) Empirical mode decomposition pre-process for higher accuracy hyperspectral image classification. IEEE International Geoscience and Remote Sensing Symposium, Boston, 7-11 July 2008, 939-941.

[9] Wu, Z.-H. and Huang, N.E. (2005) Ensemble empirical mode decomposition: A noise assisted data analysis method. Center for Ocean-Land-Atmosphere Studies, Calverton.

[10] Huang, N.E., Wu, M.-L., Long, S.R., Shen, S.S.P., Qu, W.-D., Gloersen, P. and Fan, K.L. (2003) A confidence limit for the empirical mode decomposition and hilbert spectral analysis. Proceeding Royal Society London, 459, 2317-2345.

[11] Huang, T.-L., Ren, W.-X. and Lou, M.-L. (2008) The orthogonal hilbert huang transform and its application in earthquake motion recordings analysis. 14th World Conference on Earthquake Engineering, Beijing, 12-17 October 2008. http://www.iitk.ac.in/nicee/wcee/article/14_02-0059.pdf

[12] Li, H., Wang, Y.-C. and Ma, Y.-F. (2010) Ensemble empirical mode decomposition and hilbert-huang transform applied to bearing fault diagnosis. IEEE 3rd International Congress on Image and Signal Processing, Yantai, 16-18 October 2010, 3413-3417.

[13] Golz, M., Sommer, D., Chen, M., Mandic, D. and Trustschel, U. (2007) Feature fusion for the detection of microsleep events. Journal of VLSI Signal Processing, 49, 329-342. doi:10.1007/s11265-007-0083-4

[14] Rutkowski, T.M., Cichocki, A. and Mandic, D.P. (2008) Information fusion for perceptual feedback: A brain activity sonification approach. In: Mandic, D., Golz, M., Kuh, A., Obradovic, D. and Tanaka, T., Eds., Signal Processing Techniques for Knowledge Extraction and Information Fusion, Springer, New York, 2008, 261-273. doi:10.1007/978-0-387-74367-7_14

[15] Huang, N.E., Shen, Z. and Long, S.R. (1999) A new view of nonlinear water waves: The hilbert spectrum. 
Annual Review of Fluid Mechanics, 31, 417-457. doi:10.1146/annurev.fluid.31.1.417

[16] Huang, N.E., Wu, Z.-H., Long, S.R., Arnold, K.C., Chen, X.-Y. and Blank, K. (2009) On instantaneous frequency. Advances in Adaptive Data Analysis, 1, 177-229. doi:10.1142/S1793536909000096

[17] Rehman, N. and Mandic, D.P. (2009) Multivariate empirical mode decomposition. Proceeding of the Royal Society, 466, 1291-1302.
[18] Ur Rehman, N. and Mandic, D.P. (2010) Empirical mode decomposition for trivatiate signals. IEEE Transactions on Signal Processing, 58, 1059-1068. doi:10.1109/TSP.2009.2033730

[19] Altaf, M.U.B., Gautama, T., Tanaka, T. and Mandic, D.P. (2007) Rotation invariant complex empirical mode decomposition.

http://www.commsp.ee.ic.ac.uk/ mandic/research/RI C EMD_ICASSP_2007.pdf 